\title{
ON SYMMETRIC IDEALS OF MULTILINEAR MAPPINGS BETWEEN BANACH SPACES
}

\author{
GERALDO BOTELHO ${ }^{\complement 2}$ and DANIEL M. PELLEGRINO
}

(Received 18 August 2004; revised 18 April 2005)

Communicated by A. J. Pryde

\begin{abstract}
In this paper we provide examples and counterexamples of symmetric ideals of multilinear mappings between Banach spaces and prove that if $\mathcal{I}_{1}, \ldots, \mathcal{I}_{n}$ are operator ideals, then the ideals of multilinear mappings $\mathcal{L}\left(\mathcal{I}_{1}, \ldots, \mathcal{I}_{n}\right)$ and $\left.\mid \mathcal{I}_{1}, \ldots, \mathcal{I}_{n}\right]$ are symmetric if and only if $\mathcal{I}_{1}=\cdots=\mathcal{I}_{n}$.

2000 Mathematics subject classification: primary 46G25; secondary 47B 10.

Key'ords and phrases: Banach spaces, ideals of multilinear mappings, factorization method, linearization method.
\end{abstract}

\section{Introduction}

The notion of ideal of multilinear mappings between Banach spaces goes back to Pietsch [8]. In [4], Floret-García introduced the notion of symmetric ideals of multilinear mappings, which plays an important role in the interplay between ideals of multilinear mappings and ideals of homogeneous polynomials. The aim of this paper is to provide a number of examples and counterexamples of symmetric ideals and to investigate the symmetry of the ideals $\mathcal{L}\left(\mathcal{I}_{1}, \ldots, \mathcal{I}_{n}\right)$ and $\left[\mathcal{I}_{1}, \ldots, \mathcal{I}_{n}\right]$, where each $\mathcal{I}_{j}$ is an operator ideal, which are generated by the factorization and the linearization methods.

Throughout this paper $n$ is a positive integer, $E, E_{1}, \ldots, E_{n}, F, G, G_{1}, \ldots, G_{n}$ and $H$ will stand for (real or complex) Banach spaces. The Banach space of all continuous $n$-linear mappings $A: E_{1} \times \cdots \times E_{n} \rightarrow F$ will be denoted by $\mathcal{L}\left(E_{1}, \ldots, E_{n} ; F\right)$ (and $\mathcal{L}\left({ }^{n} E ; F\right)$ if $E_{1}=\cdots=E_{n}=E$ ). For the general theory of multilinear mappings we refer to Dineen [3].

The authors were partially supported by Instituto do Milênio, IMPA and CNPq/FAPESQ.

(C) 2006 Australian Mathematical Society 1446-7887/06 \$A2.00+0.00 


\section{Symmetric ideals of multilinear mappings}

Given $n \in \mathbb{N}$, an ideal of $n$-linear mappings $\mathcal{M}$ is a subclass of the class of all continuous $\boldsymbol{n}$-linear mappings between Banach spaces such that for Banach spaces $E_{1}, \ldots, E_{n}$ and $F$, the components $\mathcal{M}\left(E_{1}, \ldots, E_{n}, F\right):=\mathcal{L}\left(E_{1}, \ldots, E_{n}, F\right) \cap \mathcal{M}$ satisfy:

(i) $\mathcal{M}\left(E_{1}, \ldots, E_{n}, F\right)$ is a linear subspace of $\mathcal{L}\left(E_{1}, \ldots, E_{n}, F\right)$ that contains the $n$-linear mappings of finite type.

(ii) The ideal property; if $A \in \mathcal{M}\left(E_{1}, \ldots, E_{n}, F\right), t \in \mathcal{L}(F, H)$ and $u_{j} \in$ $\mathcal{L}\left(G_{j}, E_{j}\right)$ for $j=1,2, \ldots, n$, then the composition $t A\left(u_{1}, \ldots u_{n}\right)$ is in $\mathcal{M}\left(G_{1}, \ldots, G_{n}, H\right)$.

By $A_{S}$ we denote the symmetrization of the $n$-linear mapping $A \in \mathcal{L}\left({ }^{n} E, F\right)$ (see, for example, [3, page 6]). According to Floret-García [4], an ideal of $n$-linear mappings $\mathcal{M}$ is said to be symmetric if $A_{S} \in \mathcal{M}\left({ }^{n} E, F\right)$ whenever $A \in \mathcal{M}\left({ }^{n} E, F\right)$.

Let us fix some terminology in order to make the interplay with the theory of ideals of polynomials clear. For $A \in \mathcal{L}\left({ }^{n} E, F\right)$, we define $\hat{A}(x):=A(x, \ldots, x)$; and given a continuous $n$-homogeneous polynomial $P: E \rightarrow F, \check{P}$ denotes the unique symmetric $n$-linear mapping associated to $P$. Given an ideal of $n$-linear mappings $\mathcal{M}$, it is easy to see that the classes $\mathcal{M}^{\vee}:=\{P: \check{P} \in \mathcal{M}\}$ and $\mathcal{M}^{\wedge}:=\{\hat{A}: A \in \mathcal{M}\}$ are ideals of $n$-homogeneous polynomials (compare with [4, Section 1.8]). It is also easy to check that $\mathcal{M}^{\vee} \subseteq \mathcal{M}^{\wedge}$ for every $\mathcal{M}$ and that $\mathcal{M}$ is symmetric if and only if $\mathcal{M}^{\vee}=\mathcal{M}^{\wedge}$.

\section{Examples}

3.1. Routine computations show that the following standard ideals are symmetric: $n$-linear mappings of finite type, approximable, nuclear, compact, and weakly compact $n$-linear mappings. Alencar [1, Corollary 3] proved that the ideal of Pietsch integral $n$-linear mappings is symmetric.

3.2. A non-symmetric ideal. A bilinear mapping $A \in \mathcal{L}\left({ }^{2} E ; F\right)$ is said to be $a b$ solutely $(1 ; 1, \infty)$-summing if $\left(A\left(x_{j}, y_{j}\right)\right)_{j=1}^{\infty}$ is absolutely summable in $F$ whenever $\left(x_{j}\right)_{j=1}^{\infty}$ is weakly absolutely summable and $\left(y_{j}\right)_{j=1}^{\infty}$ is bounded in $E$. For the theory of absolutely summing multilinear mappings the reader is referred to Matos [7]. It is straightforward to check that the class of all absolutely $(1 ; 1, \infty)$-summing bilinear mappings between Banach spaces is an ideal of bilinear mappings, denoted by $\mathcal{L}_{a s(1 ; 1, \infty)}$. We see that it is not symmetric. Define

$$
A: \ell_{\infty} \times \ell_{\infty} \longrightarrow \ell_{\infty}: A(x, y):=x_{1} u(y)
$$


where $x=\left(x_{j}\right)_{j=1}^{\infty}$ and $u: \ell_{\infty} \rightarrow \ell_{\infty}$ is a chosen bounded linear operator which fails to be absolutely 1 -summing. In order to see that $A$ is absolutely $(1 ; 1, \infty)$-summing, observe that, if $x_{j}=\left(x_{j}^{i}\right)_{i=1}^{\infty} \in \ell_{\infty}$, then

$$
\sum_{j=1}^{\infty}\left\|A\left(x_{j}, y_{j}\right)\right\|=\sum_{j=1}^{\infty}\left|x_{j}^{1}\left\|u\left(y_{j}\right)\right\| \leq\|u\|\left(\sup _{j}\left\|y_{j}\right\|\right) \sum_{j=1}^{\infty}\right| x_{j}^{1} \mid<+\infty,
$$

whenever $\left(x_{j}\right)_{j=1}^{\infty}$ is absolutely weakly summable and $\left(y_{j}\right)_{j=1}^{\infty}$ is bounded in $\ell_{\infty}$. Let $A^{t} \in \mathcal{L}\left({ }^{2} \ell_{\infty} ; \ell_{\infty}\right)$ be defined by $A^{t}(x, y):=A(y, x)$. Since $u$ is not absolutely 1 -summing, choosing $y_{j}=(1,1, \ldots)$ for every $j$, we have that $\sum_{j=1}^{\infty}\left\|A^{t}\left(x_{j}, y_{j}\right)\right\|=\infty$ for some weakly summable sequence $\left(x_{j}\right)_{j=1}^{\infty}$ in $\ell_{\infty}$, which shows that $A^{t}$ is not absolutely $(1 ; 1, \infty)$-summing. Therefore, $A \in \mathcal{L}_{a s(1: 1, \infty)}\left({ }^{2} \ell_{\infty} ; \ell_{\infty}\right)$ and, since $A_{S}=\left(A+A^{t}\right) / 2$, $A_{S} \notin \mathcal{L}_{a s(1: 1, \infty)}\left({ }^{2} \ell_{\infty} ; \ell_{\infty}\right)$, proving that $\mathcal{L}_{a s(1: 1, \infty)}$ is not symmetric.

Sections 4-5 will provide many examples of symmetric and non-symmetric ideals.

\section{The factorization method}

This factorization method, along with the linearization method (see Section 5), was introduced by Pietsch [8] and has been developed by several authors since then. Given $n \in \mathbb{N}$ and operator ideals $\mathcal{I}_{1}, \ldots, \mathcal{I}_{n}$, an $n$-linear mapping $A \in \mathcal{L}\left(E_{1}, \ldots, E_{n} ; F\right)$ is said to be of type $\mathcal{L}\left(\mathcal{I}_{1}, \ldots, \mathcal{I}_{n}\right)$, in symbols $A \in \mathcal{L}\left(\mathcal{I}_{1}, \ldots, \mathcal{I}_{n}\right)\left(E_{1}, \ldots, E_{n} ; F\right)$, if there are Banach spaces $G_{1} \ldots, G_{n}$, linear operators $u_{j} \in \mathcal{I}_{j}\left(E_{j} ; G_{j}\right), j=$ $1, \ldots, n$, and a continuous $n$-linear mapping $B \in \mathcal{L}\left(G_{1}, \ldots, G_{n} ; F\right)$ such that $A=$ $B \circ\left(u_{1}, \ldots, u_{n}\right)$. The proof that $\mathcal{L}\left(\mathcal{I}_{1}, \ldots, \mathcal{I}_{n}\right)$ is an ideal of $n$-linear mappings can be found in $[2,5]$.

THEOREM 4.1. Let $\mathcal{I}_{1}, \ldots, \mathcal{I}_{n}$ be operator ideals. The following statements are equivalent:

(a) $\mathcal{L}\left(\mathcal{I}_{1}, \ldots, \mathcal{I}_{n}\right)$ is a symmetric ideal of $n$-linear mappings.

(b) $\mathcal{L}\left(\mathcal{I}_{1}, \ldots, \mathcal{I}_{n}\right)=\mathcal{L}\left(\mathcal{I}_{\sigma(1)}, \ldots, \mathcal{I}_{\sigma(n)}\right)$ for every permutation $\sigma$ of the set $\{1, \ldots, n\}$.

(c) $\mathcal{I}_{1}=\mathcal{I}_{2}=\cdots=\mathcal{I}_{n}$.

PROOF. (c) implies (b). This is obvious.

(b) implies (a). Let $S_{n}$ denote the group of permutations of $\{1,2, \ldots, n\}$. Let $A \in \mathcal{L}\left(\mathcal{I}_{1}, \ldots, \mathcal{I}_{n}\right)\left({ }^{n} E ; F\right), A=B \circ\left(u_{1}, \ldots, u_{n}\right)$ with $u_{j} \in \mathcal{I}_{j}\left(E ; G_{j}\right), j=1, \ldots, n$, and $B \in \mathcal{L}\left(G_{1}, \ldots, G_{n} ; F\right)$. Given $\sigma \in S_{n}$, define $A_{\sigma}: E \times \cdots \times E \rightarrow F$ and $B_{\sigma}: G_{\sigma^{-1}(1)} \times \cdots \times G_{\sigma^{-1}(n)} \rightarrow F$ by

$$
\begin{aligned}
& A_{\sigma}\left(x_{1}, \ldots, x_{n}\right):=A\left(x_{\sigma(1)}, \ldots, x_{\sigma(n)}\right), \\
& B_{\sigma}\left(y_{1}, \ldots, y_{n}\right):=B\left(y_{\sigma(1)}, \ldots, y_{\sigma(n)}\right),
\end{aligned}
$$


to get $A_{\sigma}=B_{\sigma} \circ\left(u_{\sigma^{-1}(1)}, \ldots, u_{\sigma^{-1}(n)}\right)$. This proves that, for every $\sigma \in S_{n}$,

$$
A_{\sigma} \in \mathcal{L}\left(\mathcal{I}_{\sigma^{-1}(1)}, \ldots, \mathcal{I}_{\sigma^{-1}(n)}\right)\left({ }^{n} E ; F\right)=\mathcal{L}\left(\mathcal{I}_{1}, \ldots, \mathcal{I}_{n}\right)\left({ }^{n} E ; F\right) .
$$

Since $n ! A_{S}=\sum_{\sigma \in S_{n}} A_{\sigma}$ it follows that $A_{S} \in \mathcal{L}\left(\mathcal{I}_{1}, \ldots, \mathcal{I}_{n}\right)\left({ }^{n} E ; F\right)$.

(a) imples (c). Select $i, j \in\{1,2, \ldots, n\}$ and let $u \in \mathcal{I}_{i}(E ; F)$. Fix $\varphi \in E^{\prime}, a \in E$ such that $\varphi(a)=1$ and define $A \in \mathcal{L}\left({ }^{n} E ; F\right), B \in \mathcal{L}(\mathbb{K}, \ldots, F, \ldots, \mathbb{K} ; F)$ by

$$
\begin{aligned}
& A\left(x_{1}, \ldots, x_{n}\right):=\varphi\left(x_{1}\right) \cdots \widehat{\varphi\left(x_{i}\right)} \cdots \varphi\left(x_{n}\right) u\left(x_{i}\right), \\
& B\left(\lambda_{1}, \ldots, \lambda_{i-1}, z, \lambda_{i+1}, \ldots, \lambda_{n}\right):=\lambda_{1} \cdots \widehat{\lambda_{i}} \cdots \lambda_{n} z,
\end{aligned}
$$

where $\widehat{\alpha_{i}}$ means that $\alpha_{i}$ is omitted. Thus $A=B \circ(\varphi, \ldots, u, \ldots, \varphi)$, proving that $A \in \mathcal{L}\left(\mathcal{I}_{1}, \ldots, \mathcal{I}_{n}\right)\left({ }^{n} E ; F\right)$. By assumption it follows that $A_{S} \in \mathcal{L}\left(\mathcal{I}_{1}, \ldots, \mathcal{I}_{n}\right)\left({ }^{n} E ; F\right)$, say $A_{S}=C \circ\left(v_{1}, \ldots, v_{n}\right)$ with $v_{j} \in \mathcal{I}_{j}\left(E ; G_{j}\right), j=1, \ldots, n$, and $C \in \mathcal{L}\left(G_{1}, \ldots\right.$, $\left.G_{n} ; F\right)$. If $C_{L}: G_{1} \hat{\otimes}_{\pi} \cdots \hat{\otimes}_{\pi} G_{n} \rightarrow F$ is the linearization of $C$ and $i_{j}: G_{j} \rightarrow$ $G_{1} \hat{\otimes}_{\pi} \cdots \hat{\otimes}_{\pi} G_{n}$ is the operator defined by

$$
i_{j}(z):=v_{1}(a) \otimes \cdots \otimes v_{j-1}(a) \otimes z \otimes v_{j+1}(a) \otimes \cdots \otimes v_{n}(a),
$$

there are nonzero constants $K_{1}$ and $K_{2}$ such that $C_{L} \circ i_{j} \circ v_{j}=K_{1} u+K_{2} \varphi(\cdot) u(a)$. Since $v_{j} \in \mathcal{I}_{j}\left(E ; G_{j}\right)$ and $K_{2} \varphi(\cdot) u(a)$ is a finite rank operator, it follows that $u \in \mathcal{I}_{j}(E ; F)$, proving that $\mathcal{I}_{i} \subseteq \mathcal{I}_{j}$. The proof is complete because $i$ and $j$ are arbitrary.

REMARK 4.2. It is interesting that even in a symmetric ideal $\mathcal{M}$ of $n$-linear mappings it is not always true that $A \in \mathcal{M}$ whenever $A_{S} \in \mathcal{M}$. Leung [6] can be used to accomplish this task, but we describe a (simpler) counterexample: a bilinear mapping $A \in \mathcal{L}\left({ }^{2} E ; F\right)$ is said to be absolutely $(1 ; 2,2)$-summing (or 2-dominated), in symbols $A \in \mathcal{L}_{a s(1 ; 2,2)}\left({ }^{2} E ; F\right)$, if $\left(A\left(x_{j}, y_{j}\right)\right)_{j=1}^{\infty}$ is absolutely summable in $F$ whenever $\left(x_{j}\right)_{j=1}^{\infty}$ and $\left(y_{j}\right)_{j=1}^{\infty}$ are weakly 2-summable in $E$. It is well known that $\mathcal{L}_{a s(1 ; 2,2)}=\mathcal{L}\left(\Pi_{2}, \Pi_{2}\right)$ (see, for example, [9, Proposition 3.6]), where $\Pi_{2}$ is the ideal of all absolutely 2summing operators. So, from Theorem 4.1 , it follows that $\mathcal{L}_{a s(1 ; 2,2)}$ is a symmetric ideal of bilinear mappings. Consider the bilinear mapping $A \in \mathcal{L}\left({ }^{2} \ell_{2} ; \ell_{1}\right)$ given by

$$
A\left(\left(\alpha_{i}\right)_{i=1}^{\infty},\left(\beta_{i}\right)_{i=1}^{\infty}\right):=\left(\alpha_{1} \beta_{2}-\alpha_{2} \beta_{1}, \alpha_{3} \beta_{4}-\alpha_{4} \beta_{3}, \alpha_{5} \beta_{6}-\alpha_{6} \beta_{5}, \ldots\right) .
$$

If $\left(e_{j}\right)_{j=1}^{\infty}$ are the canonical unit vectors of $\ell_{2}$, we have that $A\left(e_{2 j+1}, e_{2 j+2}\right)=e_{j+1}$ for every $j \in \mathbb{N}$. Then $A \notin \mathcal{L}_{a s(1 ; 2,2)}\left({ }^{2} \ell_{2} ; \ell_{1}\right)$ (because $\left(e_{j}\right)_{j=1}^{\infty}$ is weakly 2 -summable in $\left.\ell_{2}\right)$, but $A_{S} \in \mathcal{L}_{a s(1 ; 2,2)}\left({ }^{2} \ell_{2} ; \ell_{1}\right)$ (because $A_{S} \equiv 0$ ).

\section{The linearization method}

Let the notation . $[$. . mean that the $i$-th coordinate is not involved. For $i=1, \ldots, n$, let $I_{i}: \mathcal{L}\left(E_{1}, \ldots, E_{n} ; F\right) \rightarrow \mathcal{L}\left(E_{i} ; \mathcal{L}\left(E_{1}, .|i|,, E_{n} ; F\right)\right)$ be the isometric isomorphism 
defined by $I_{i}(A)\left(x_{i}\right)\left(x_{1}, . ! 1, x_{n}\right):=A\left(x_{1}, \ldots, x_{n}\right)$. Of course, if $E_{1}=\cdots=E_{n}$ and $A$ is symmetric, then $I_{1}(A)=I_{2}(A)=\cdots=I_{n}(A)$. In this case we write $I(A)$ instead of $I_{i}(A)$. An $n$-linear mapping $A \in \mathcal{L}\left(E_{1}, \ldots, E_{n} ; F\right)$ is said to be of type $\left[\mathcal{I}_{1}, \ldots, \mathcal{I}_{n}\right]$, in symbols $A \in\left[\mathcal{I}_{1}, \ldots, \mathcal{I}_{n}\right]\left(E_{1}, \ldots, E_{n} ; F\right)$, if, for every $i=1, \ldots, n$, the operator $I_{i}(A) \in \mathcal{I}_{i}\left(E_{i} ; \mathcal{L}\left(E_{1},{ }_{i}{ }^{[i]} ., E_{n} ; F\right)\right)$. Again, the proof that $\left[\mathcal{I}_{1}, \ldots, \mathcal{I}_{n}\right]$ is an ideal of $n$-linear mappings can be found in [2] and [5].

LEMMA 5.1. If $\mathcal{I}_{1}$ and $\mathcal{I}_{2}$ are operator ideals such that $\left[\mathcal{I}_{1}, \mathcal{I}_{2}\right]$ is a symmetric ideal of bilinear mappings, then $\mathcal{I}_{1}=\mathcal{I}_{2}$.

ProOF. Let $u \in \mathcal{I}_{1}(E ; F)$. Fix $\varphi \in E^{\prime}, a \in E$ such that $\varphi(a)=1$ and define $A \in \mathcal{L}\left({ }^{2} E ; F\right), T \in \mathcal{L}(F ; \mathcal{L}(E ; F))$ by $A(x, y):=\varphi(y) u(x)$ and $T(z)(y):=\varphi(y) z$. It follows that $T \circ u=I_{1}(A)$, hence $I_{1}(A) \in \mathcal{I}_{1}(E ; \mathcal{L}(E ; F))$. On the other hand, $I_{2}(A)=\varphi(\cdot) u$, which is a finite rank operator, therefore $A \in\left[\mathcal{I}_{1}, \mathcal{I}_{2}\right]\left({ }^{2} E ; F\right)$. By assumption we have $A_{S} \in\left[\mathcal{I}_{1}, \mathcal{I}_{2}\right]\left({ }^{2} E ; F\right)$. Since $2 I\left(A_{S}\right)=I_{1}(A)+I_{2}(A)$, it follows that $I_{1}(A) \in \mathcal{I}_{2}(E ; \mathcal{L}(E ; F))$. Defining $U: \mathcal{L}(E ; F) \rightarrow F$ by $U(v):=v(a)$, we get $U \circ I_{1}(A)=u$, which proves that $u \in \mathcal{I}_{2}(E ; F)$. We proved that $\mathcal{I}_{1} \subseteq \mathcal{I}_{2}$ and the other inclusion is analogous.

THEOREM 5.2. Let $\mathcal{I}_{1}, \ldots, \mathcal{I}_{n}$ be operator ideals. The following statements are equivalent:

(a) $\left[\mathcal{I}_{1}, \ldots, \mathcal{I}_{n}\right]$ is a symmetric ideal of $n$-linear mappings.

(b) $\left[\mathcal{I}_{1}, \ldots, \mathcal{I}_{n}\right]=\left[\mathcal{I}_{\sigma(1)}, \ldots, \mathcal{I}_{\sigma(n)}\right]$ for every permutation $\sigma$ of the set $\{1,2, \ldots, n\}$.

(c) $\mathcal{I}_{1}=\mathcal{I}_{2}=\cdots=\mathcal{I}_{n}$.

ProOF. (c) implies (b). This is obvious.

(b) implies (a). Let $A \in\left[\mathcal{I}_{1}, \ldots, \mathcal{I}_{n}\right]\left({ }^{n} E ; F\right)$. Then $A \in\left[\mathcal{I}_{\sigma(1)}, \ldots, \mathcal{I}_{\sigma(n)}\right]\left({ }^{n} E ; F\right)$ for every $\sigma$ by assumption; hence $I_{j}(A) \in \mathcal{I}_{k}\left(E ; \mathcal{L}\left({ }^{n-1} E ; F\right)\right.$ ) for every $j, k=$ $1, \ldots, n$. Fix a permutation $\sigma$ and consider the operator $R_{o}: \mathcal{L}\left({ }^{n-1} E ; F\right) \rightarrow$ $\mathcal{L}\left({ }^{n-1} E ; F\right)$ defined by $R_{\sigma}(B)\left(x_{2}, \ldots, x_{n}\right):=B\left(x_{\sigma(1)},{ }^{\left[\sigma^{-1}(1)\right]}, x_{\sigma(n)}\right)$. where ${ }^{\left|\sigma_{\ldots}^{-1}(!)\right|}$ means that the $\sigma^{-1}(1)$-th coordinate is omitted. For every $x_{1}, \ldots, x_{n} \in E$,

$$
\begin{aligned}
n ! I\left(A_{S}\right)\left(x_{1}\right)\left(x_{2}, \ldots, x_{n}\right) & =\sum_{\sigma \in S_{n}} A\left(x_{\sigma(1)}, \ldots, x_{\sigma(n)}\right) \\
& =\sum_{\sigma \in S_{n}} I_{\sigma^{-1}(1)}(A)\left(x_{1}\right)\left(x_{\sigma(1)},{ }^{\mid \sigma^{-1}(1) !}, x_{\sigma(n)}\right) \\
& =\sum_{\sigma \in S_{n}} R_{\sigma} \circ I_{\sigma^{-1}(1)}(A)\left(x_{1}\right)\left(x_{2}, \ldots, x_{n}\right),
\end{aligned}
$$

proving that $n ! l\left(A_{S}\right)=\sum_{\sigma \in S_{n}} R_{\sigma} \circ I_{\sigma^{-1}(1)}(A)$. However, $I_{\sigma^{-1}(1)}(A)$ belongs to $\mathcal{I}_{k}$ for every $\sigma$ and every $k$, so $I\left(A_{S}\right) \in \mathcal{I}_{k}\left(E ; \mathcal{L}\left({ }^{n-1} E ; F\right)\right)$ for every $k$. It follows that $A_{S} \in\left[\mathcal{I}_{1}, \ldots, \mathcal{I}_{n}\right]\left({ }^{n} E ; F\right)$. 
(a) implies (c). By Lemma 5.1, we may assume $n \geq 3$. Fix $j \in\{2,3, \ldots, n\}$ and let $T \in\left[\mathcal{I}_{1}, \mathcal{I}_{j}\right]\left({ }^{2} E ; F\right)$. As before, fix $\varphi \in E^{\prime}, a \in E$ such that $\varphi(a)=1$ and define $A \in \mathcal{L}\left({ }^{n} E ; F\right), \psi_{1}: \mathcal{L}(E ; F) \rightarrow \mathcal{L}\left({ }^{n-1} E ; F\right)$ by

$$
\begin{aligned}
A\left(x_{1}, \ldots, x_{n}\right) & :=\varphi\left(x_{2}\right) \cdots \widehat{\varphi\left(x_{j}\right)} \cdots \varphi\left(x_{n}\right) T\left(x_{1}, x_{j}\right), \\
\psi_{1}(S)\left(x_{2}, \ldots, x_{n}\right) & =\varphi\left(x_{2}\right) \cdots \widehat{\varphi\left(x_{j}\right)} \cdots \varphi\left(x_{n}\right) S\left(x_{j}\right),
\end{aligned}
$$

where $\widehat{\varphi\left(x_{j}\right)}$ means that $\varphi\left(x_{j}\right)$ is omitted. From $I_{1}(A)=\psi_{1} \circ I_{1}(T)$ we get $I_{1}(A) \in$ $\mathcal{I}_{1}\left(E ; \mathcal{L}\left({ }^{n-1} E ; F\right)\right)$. Defining $U: \mathcal{L}\left({ }^{n-1} E ; F\right) \rightarrow \mathcal{L}\left({ }^{n-1} E ; F\right)$ by

$$
U(B)\left(x_{2}, \ldots, x_{n}\right):=B\left(x_{3}, \ldots, x_{j-1}, x_{2}, x_{j}, x_{j+1}, \ldots, x_{n}\right),
$$

it follows that $I_{j}(A)=U \circ \psi_{1} \circ I_{2}(T)$, hence $I_{j}(A) \in \mathcal{I}_{j}\left(E ; \mathcal{L}\left({ }^{n-1} E ; F\right)\right)$. It is not difficult to see that for $k=2,3, \ldots, n, k \neq j, I_{k}(A)$ is a finite rank operator and so $A \in\left[\mathcal{I}_{1}, \ldots, \mathcal{I}_{n}\right]\left({ }^{n} E ; F\right)$. By assumption we have that $A_{S} \in\left[\mathcal{I}_{1}, \ldots, \mathcal{I}_{n}\right]\left({ }^{n} E ; F\right)$ also. Using that

$$
\begin{aligned}
A_{S}\left(x_{1}, \ldots, x_{n}\right)= & \frac{1}{n !} \sum_{\sigma \in S_{n}} A\left(x_{\sigma(1)}, \ldots, x_{\sigma(n)}\right) \\
= & \frac{1}{2 n !} \sum_{\sigma \in S_{n}}\left(A\left(x_{\sigma(1)}, x_{\sigma(2)}, \ldots, x_{\sigma(n)}\right)\right. \\
& \left.+A\left(x_{\sigma(j)}, x_{\sigma(2)}, \ldots, x_{\sigma(1)}, \ldots, x_{\sigma(n)}\right)\right) \\
= & \frac{1}{2 n !} \sum_{\sigma \in S_{n}} \varphi\left(x_{\sigma(2)}\right) \cdots \widehat{\varphi\left(x_{\sigma(j)}\right)} \cdots \varphi\left(x_{\sigma(n)}\right) \\
& \times\left(T\left(x_{\sigma(1)}, x_{\sigma(j)}\right)+T\left(x_{\sigma(j)}, x_{\sigma(1)}\right)\right) \\
= & \frac{1}{n !} \sum_{\sigma \in S_{n}} \varphi\left(x_{\sigma(2)}\right) \cdots \widehat{\varphi\left(x_{\sigma(j)}\right)} \cdots \varphi\left(x_{\sigma(n)}\right) T_{S}\left(x_{\sigma(1)}, x_{\sigma(j)}\right),
\end{aligned}
$$

and defining $\Delta_{n}:=\left\{\sigma \in S_{n}: \sigma(1)=1\right.$ or $\left.\sigma(j)=1\right\}$, it follows that

$$
\begin{aligned}
n ! I\left(A_{S}\right) & \left(x_{1}\right)\left(x_{2}, \ldots, x_{n}\right) \\
= & \sum_{\sigma \in \Delta_{n}} \varphi\left(x_{\sigma(2)}\right) \cdots \widehat{\varphi\left(x_{\sigma(j)}\right)} \cdots \varphi\left(x_{\sigma(n)}\right) I\left(T_{S}\right)\left(x_{\sigma(1)}\right)\left(x_{\sigma(j)}\right) \\
& +\sum_{\sigma \notin \Delta_{n}} \varphi\left(x_{\sigma(2)}\right) \cdots \widehat{\varphi\left(x_{\sigma(j)}\right)} \cdots \varphi\left(x_{\sigma(n)}\right) T_{S}\left(x_{\sigma(1)}, x_{\sigma(j)}\right) .
\end{aligned}
$$

Therefore for each $\sigma \notin \Delta_{n}, \sigma(1) \neq 1$, and $\sigma(j) \neq 1$,

$$
V\left(x_{1}\right)\left(x_{2}, \ldots, x_{n}\right):=\sum_{\sigma \notin \Delta_{n}} \varphi\left(x_{\sigma(2)}\right) \cdots \widehat{\varphi\left(x_{\sigma(j)}\right)} \cdots \varphi\left(x_{\sigma(n)}\right) T_{S}\left(x_{\sigma(1)}, x_{\sigma(j)}\right)
$$


is a finite rank operator in $\mathcal{L}\left(E ; \mathcal{L}\left({ }^{n-1} E ; F\right)\right)$. For every $k=1, \ldots, n, n ! I\left(A_{S}\right) \in$ $\mathcal{I}_{k}\left(E ; \mathcal{L}\left({ }^{n-1} E ; F\right)\right)$, then the operator $\left.R: E \rightarrow \mathcal{L}\left({ }^{n-1} E ; F\right)\right)$ defined by

$$
R\left(x_{1}\right)\left(x_{2}, \ldots, x_{n}\right):=\sum_{\sigma \in \Delta_{n}} \varphi\left(x_{\sigma(2)}\right) \cdots \widehat{\varphi\left(x_{\sigma(j)}\right)} \cdots \varphi\left(x_{\sigma(n)}\right) I\left(T_{S}\right)\left(x_{\sigma(1)}\right)\left(x_{\sigma(j)}\right)
$$

belongs to $\mathcal{I}_{k}\left(E ; \mathcal{L}\left({ }^{n-1} E ; F\right)\right)$ for every $k$ as well. Defining $f: \mathcal{L}\left({ }^{n-1} E ; F\right) \rightarrow$ $\mathcal{L}(E ; F)$ by $f(B)(y):=B(y, a, \ldots, a)$, there are nonzero constants $K_{1}$ and $K_{2}$ such that for every $x, y \in E$,

$$
(f \circ R)(x)(y)=K_{1} I\left(T_{S}\right)(x)(y)+K_{2} \varphi(y) I\left(T_{S}\right)(x)(a) .
$$

If $g: \mathcal{L}\left({ }^{n-1} E ; F\right) \rightarrow F$ is defined by $g(B):=B(a, \ldots, a)$, then

$$
\left(g \circ I\left(A_{S}\right)\right)(x)=\frac{1}{n} T(x, a)+\frac{1}{n} T(a, x)+\frac{n-2}{n} \varphi(x) T(a, a),
$$

for every $x \in E$. Since $g \circ I\left(A_{S}\right) \in \mathcal{I}_{k}(E ; F)$ for every $k$, and $\varphi(\cdot) T(a, a)$ is a finite rank operator, it follows that $(T(\cdot, a)+T(a, \cdot)) \in \mathcal{I}_{k}(E ; F)$ for every $k$. Finally, let $\psi_{2}: F \rightarrow \mathcal{L}(E ; F)$ be given by $\psi_{2}(z)(x):=\varphi(x) z$. Then

$$
(f \circ R)(x)(y)=K_{1} I\left(T_{S}\right)(x)(y)+\frac{K_{2}}{2} \psi_{2}(T(x, a)+T(a, x))(y),
$$

for every $x, y \in E$, which proves that

$$
f \circ R=K_{1} I\left(T_{S}\right)+\frac{K_{2}}{2}\left(\psi_{2} \circ(T(\cdot, a)+T(a, \cdot))\right) .
$$

Therefore, $I\left(T_{S}\right) \in \mathcal{I}_{k}(E ; \mathcal{L}(E ; F))$ for every $k$, and this implies that

$$
T_{S} \in\left[\mathcal{I}_{1}, \mathcal{I}_{j}\right]\left({ }^{2} E ; F\right)
$$

Thus far we have proved that $\left[\mathcal{I}_{1}, \mathcal{I}_{j}\right]$ is symmetric. Now we call on Lemma 5.1 to conclude that $\mathcal{I}_{1}=\mathcal{I}_{j}$ for $j=2,3, \ldots, n$.

Final remark The notion of strongly symmetric quasi-normed ideals of multilinear mappings was introduced by Floret-Garcia [4]. It is easy to see that, given a normed operator ideal $\mathcal{I}$, the symmetric ideals $\mathcal{L}(\mathcal{I}, \ldots, \mathcal{I})$ and $[\mathcal{I}, \ldots, \mathcal{I}]$ are strongly symmetric (see [2] for the quasi-norm on $\mathcal{L}(\mathcal{I}, \ldots, \mathcal{I})$ and the norm on $[\mathcal{I}, \ldots, \mathcal{I}]$ ).

\section{References}

[1] R. Alencar, 'On reflexivity and basis for $\mathcal{P}\left({ }^{m} E\right)$ ', Proc. Roy: Irish Acad. Sect. A 85 (1985), 131-138. 
[2] H. A. Braunss, Multi-ideals with special properties (Blätter Potsdamer Forschungen 1/87, Potsdam. 1987).

[3] S. Dineen, Complex analysis on infinite dimensional spaces (Springer, London, 1999).

[4] K. Floret and D. García, 'On ideals of polynomials and multilinear mappings between Banach spaces', Arch. Math. (Basel) 81 (2003), 300-308.

[5] S. Geiss, 'Ein Faktorisierungssatz für multilineare Funktionale', Math. Nachr. 134 (1987), 149-159.

[6] D. H. Leung, 'Some remarks on regular Banach spaces', Glasg. Math. J. 38 (1996), 243-248.

[7] M. Matos, 'Absolutely summing holomorphic mappings', An. Acad. Brasil. Ciênc. 68 (1996), 1-13.

[8] A. Pietsch, 'Ideals of multilinear functionals', in: Proceedings of the Second International Conference on Operator Algebras, Ideals and Their Applications in Theoretical Physics, Teubner-Texte Math. 67 (Teubner, Leipzig, 1983) pp. 185-199.

[9] B. Schneider, 'On absolutely $p$-summing and related multilinear mappings', Wiss. Z. Brandenburg. Landeshochsch. 35 (1991), 105-117.

Faculdade de Matemática

Univ. Federal de Uberlândia

38.400-902 Uberlândia

Brazil

e-mail: botelho@ufu.br
Departamento de Matemática e Estatística

Univ. Federal de Campina Grande

58.109-970 Campina Grande

Brazil

e-mail: dmp@dme.ufcg.edu.br 